\title{
Enhancing digital literacy with an intelligent conversational agent for senior citizens in Thailand
}

\author{
Kanyarat Sriwisathiyakun $^{1}$ (D) $\cdot$ Chawaporn Dhamanitayakul ${ }^{2}$
}

Received: 15 March 2021 / Accepted: 13 December 2021 / Published online: 5 January 2022

(c) The Author(s), under exclusive licence to Springer Science+Business Media, LLC, part of Springer Nature 2021

\begin{abstract}
Intelligent conversational agents have been implemented as virtual assistants in mobile applications to facilitate, engage, and interact with users for optimal learning experiences. With 24/7 availability, providing instant and consistent responses, chatbots, as a type of intelligent conversational agent, will help benefit the learning communication, makes the entire learning experience more engaging for the learners. They have also been successfully used by the elderly to encourage behavioral change for their intended purpose. This study investigated baseline data on the use of digital platforms of senior citizens in Bangkok Metropolitan and the six regions of Thailand and developed a chatbot from the derived data. The chatbot contained learning media and service function, served as a platform to enhance digital literacy for the senior citizens in Thailand. The study was conducted in 3 phases, the baseline survey on the use of digital platforms of the senior citizens in Thailand, the development of the chatbot and learning media, and the pre-experimental expert validation. The samples were 422 senior citizens. The data were collected by questionnaires, focused group discussion, and interviews with experts, and analyzed by percentage, mean, standard deviation, and content analysis. Results were incorporated in the design and development of the chatbot innovation following the software development life cycle (SDLC) framework. Expert feedback revealed that this chatbot innovation was easy to access, convenient to request for operations, artistically appealing, and comprehensive in content and functionality for enhancing digital literacy skills, which are to access, analyze, evaluate, participate, and act. In the next research sequence, this innovation will subsequently be experimented with more senior citizens to prepare and improve their digital competence to consequently equip them with the necessary capacities to keep up with Thailand's transition towards a full-blown aging society.
\end{abstract}

Keywords Intelligent Conversational agent $\cdot$ Chatbot $\cdot$ Digital literacy $\cdot$ Senior citizens

Kanyarat Sriwisathiyakun

Kanyarat.sr@kmitl.ac.th

1 School of Industrial Education and Technology, King Mongkut's Institute of Technology Ladkrabang, Bangkok 10520, Thailand

2 College of Communication Arts, Rangsit University, Pathum Thani 12130, Thailand 


\section{Introduction}

As Knodel et al. (2013) reported, Thailand has become the world's top-three aging society with over 13 million ( $20 \%$ of its citizens) who are at least 60 years old. Furthermore, the figure was anticipated to exceed 20 million (35.8\%) in 2040. This notion presents an alarming implication that a Thai senior citizen will be walking among every three non-senior Thais.

As a result of the aforementioned situation, Thailand has defined the 20-year Thai National Strategy (2017-2036) and the 2nd Thai National Plan for the Elderly (2002-2021), which focuses on promoting health knowledge, basic self-prevention and care, as well as strengthening the elderly's organization. Meanwhile, Thailand now confronts a shortage of personnel with appropriate expertise and experience in caring for the elderly to cope with the increasing senior population. Therefore, it is a challenge for research and development of Intelligent conversational agents or chatbots to facilitate and improve the quality of life of the elderly (SD Thailand, 2019).

Moreover, under the uncertain COVID-19 situation, Thai people have not been satisfied with the experience with service providers by personnel yet and conventional service provided via phone calls may be insufficient for people who have high concerns about their service information. To solve these limitations, AI technology, particularly a chatbot, was considered because of its ability to provide practically instant and up-to-date answers to almost every question a person could ask (Rodsawang et al., 2020).

Intelligent conversational agents are a form of artificial intelligence (AI) with capacities to evolve via machine learning. This digital invention creates a fake human interlocutor with abilities to predict and generate conversational reactions based on previously gathered knowledge (Vaidyam et al., 2019). While intelligent conversational agents refer to a larger scope, chatbots can be categorized as a type/an example of all available intelligent conversational agents.

The emergence of today's digital world introduces global impacts to most ways of life. Hence, apart from real-world competencies, senior citizens also need digital literacy to survive, e.g., personal safeguarding, protection of data, selection of digital tools, and access to online information. Consequently, digital literacy is a vital quality for today's senior citizens. Digital literacy training and support should be provided so that this category of citizens could learn and live safely in this digital era.

Based on the introduced circumstance, if chatbots could enhance digital literacy for senior citizens in Thailand, their living quality and preparedness might improve. This study began by investigating baselines on the use of digital platforms of senior citizens in Bangkok Metropolitan and the six regions of Thailand. Subsequently, the obtained data were incorporated to design and innovate a chatbot, serving as a platform for these senior citizens to access media and content to enhance digital literacy skills and maximize the use of digital devices in their possessions. This study was projected to offer senior citizens more opportunities to learn, reinforce self-esteem, strengthen overall social wellbeing, and maintain self-development by alleviating digital competence. These benefits could substantially help senior citizens reduce gaps and mitigate challenges as Thailand rapidly approaches an aging society. 


\section{Literature review}

A systematic literature review based on Kitchenham (2004)'s procedure was used to review literature related to the topic of digital literacy for senior citizens and intelligent conversational agents. The review protocol was created with the review question and review strategy as a fundamental element. The strategy was to search for digital publications using terms like "digital literacy", "senior citizens", "intelligent conversational agent", and "education", among others, that were relevant to the research issue. For this review, articles pertaining to the research subject that were published between 2016 and 2021 were chosen. The review questions included questions regarding the principles and value of digital literacy for senior citizens, as well as questions about the concepts and trends of using intelligent conversational agents or chatbots in education and assisting the senior citizens in self-improvement, particularly in Thailand. Articles with consistent content and responses to review questions were extracted, synthesized, and compiled as in following sequence.

\subsection{Digital literacy for senior citizens}

When it comes to digital devices, it mainly refers to smartphones, tablets, and personal computers. According to a survey by Sindecharak and Kwanyoo (2020), the majority of the early seniors aged 60-69 years use smartphones, followed by feature phones, tablets, and personal computers, while the mid- and late-elderly groups use the most feature phone handsets. Digital literacy refers to abilities gained from knowledge and skills to operate digital devices and often refers to an Information and Communications Technology (ICT) skill set, which is highly essential in today's computerized world (Deursen et al., 2016; Friemel, 2016). Furthermore, digital literacy was defined as a set of skills for searching, evaluating, creating, and communicating with digital information, technology, and media. The most fundamental level is described as the use of digital technology in everyday life, such as searching for information and communicating (Techataweewan \& Prasertsin, 2018). Based on the findings of Dhamanitayakul (2017)'s study, Table 1 illustrates the expected attributes of digital literacy skill and indicators.

Table 1 Guidelines for Enhancing Digital literacy for Senior citizens according to Expected Attributes and Indicators

\begin{tabular}{ll}
\hline Digital Literacy Skill & Indicators \\
\hline $\begin{array}{l}\text { Access } \\
\text { Analyze \& Evaluate }\end{array}$ & $\begin{array}{l}\text { Knowledge of how to access correct information } \\
\text { benefits needed } \\
\text { Ability to evaluate, classify, and apply types of information properly }\end{array}$ \\
Create & $\begin{array}{l}\text { Knowledge and ability to participate in the society in a beneficial way for } \\
\text { themselves and for the society through digital media or devices } \\
\text { knowledge and ability in creating changes or acts that are beneficial for the } \\
\text { sct }\end{array}$ \\
& society through digital media or devices \\
\hline
\end{tabular}


Sultana (2018) indicated that digital literacy deals more with the use of such digital devices to connect with others in social networks than for computation and suggested that it takes not only familiarity with software and devices but also social ethics to become digitally literate. The absence of digital literacy was among commonly identified barriers preventing senior citizens from maximizing internet utilization. Senior citizens recognize their age as a factor in the adoption of technology. A lack of skills and limited social and institutional support make it difficult for them to gain experience and comfort with technology. However, support systems, such as family and peers, can help mediate senior citizens' reluctance with technology (Schreurs et al., 2017). Nevertheless, since the internet has become a fundamental drive in Thai society, it would be wise for the Thai citizens to equip themselves with the following digital literacy skills that include using media in digital formats such as Social media, email, online transaction, and websites, as well as accessing other forms of media, such as videos and chatting safely. To bridge the digital literacy gap, Thailand has made an attempt to develop a nationwide force of digital literacy trainers, with at least one trainer teaching people the basics of digital literacy in each community (The Nation Thailand, 2017). Therefore, to assist in the resolution of the digital literacy gap, research and development to apply chatbot technology to digital literacy training is highly attractive.

\subsection{Intelligent Conversational Agents}

Intelligent conversational agents are classified as natural-language user interfaces with capacities to serve as virtual interlocutors in chatbot-human interactions. Chatbots, as one class of intelligent conversational agents, often embedded in online messaging applications, are AI conversational software that use a natural language to simulate responsive dialogues in conversations with humans in forms of voices and texts to offer conversational convenience (Shevat, 2017; Liao et al., 2018; Almurtadha, 2019; Oxford Dictionary, 2019; Luxton \& Sirotin, 2020). Hence, chatbots have been implemented as virtual assistants in mobile applications to facilitate, engage, and interact with users for optimal learning experiences.With 24/7 availability, providing instant and consistant responses, chatbots will help benefit the learning communication, makes the entire learning experience more engaging for the learners.

Reviewing recent studies on intelligent conversational agent, often referred to as conversational agent or chatbot, in areas related to education and a wide range of conversational agent applications have shown the potential of a conversational agent in support of teaching and learning. According to the current landscape of chatbots in education, chatbots are most commonly used for student skill development purposes. In the pedagogical roles, chatbots have been used as educational tools to improve skills and motivate students by supporting learning and teaching activities. Moreover, incorporating chatbots in the mentoring role, chatbots are mostly concerned with three mentoring topics 1) Self-Regulated Learning, 2) Life Skills, and 3) Learning Skills and three mentoring methods 1) Scaffolding, 2) Recommending, and 3) Informing (Wollny et al., 2021). 
Conversational agents can be beneficial in education taking the role of teachers, students, or companions according to a certain pedagogic goal pursued. For learners, conversational agent successfully provides opportunities to improve their skills with instant, content-related, and quality interactions especially in graduatelevel courses online (Pérez-Marín, 2021; Song et al., 2017). It was also found that conversational agent with the ability to provides scaffolds by voice and text when needed during an online video lecture, significantly improved meaningful learning (Winkler et al., 2020). This type of voice-based conversational agent has also been successful in encouraging the elderly to exercise for better health. The "FitChat" AI Chatbot on Mobile has been created and introduced to the elderly, the evaluation results were found that the elderly prefer voice based chat over text notifications or free text entry and that voice is a powerful mode for encouraging motivation (Wiratunga et al., 2020). In addition, Hobert and Wolff (2019) analyzed trends of research on the use of conversational agent in educational purposes. They can observe an upward trend towards using mobile messenger-like conversational agents in education, including intelligent chatbots receive more attention in research, whereas the trend of using embodied conversational agents seems to be decreasing. Their analysis of the didactical perspective also shows that pedagogical conversational agents are most often designed for non-formal learning settings. Students will learn by interacting with conversational agents via mobile or webbased application anywhere and anytime.

Instead of providing graphical user interface (GUI), chatbot communicates with user via conversational user interface (CUI) or integration with existing messaging applications (Mislevics et al., 2018). Chatbot is responsible for understanding the messages that convey a user's needs and creating a response that meets that need. In general, chatbot architecture has a User Interface Component for receiving the user's request through an application using text or speech input, such as a messenger application like Line application, Facebook Messenger, WhatsApp, WeChat, etc. And then, the user messages will be forwarded to User Message Analysis Component. In this component, The user's language is identified and translated into the language of the chatbot's NLU (Natural Language Understanding) in order to extract their purpose, which is the "Intent", and the parameters of intent, called entities, from the user request. Also, in chatbot architecture, there is a Dialog Management Component that controls and updates the conversation context and predicts next action. The "next action" could be a response to the user with an appropriate message to inquire additional context information from the user to fulfill missing entities, or it could be retrieving the information needed to fulfill the user's intent through external APIs calls or Database requests. Once the "next action" corresponds to responding to the user, then the Response Generation Component takes over. This component generates a response message to a user using pre-defined templates with placeholders, then the appropriate message is displayed to the user and the chatbot goes into a wait mode listening for the next user input (Adamopoulou \& Moussiades, 2020; Kompella, 2018).

To build and deploy chatbot, Dialogflow on Google Cloud seems to be a very powerful platform. Dialogflow is a natural language understanding platform that makes it simple to develop and integrate a conversational user interface into any mobile app, 
web app, device, bot, interactive voice response system, and more. Dialogflow can assess a variety of user inputs, including text and audio. It can also respond to users via text or synthetic speech (Google Cloud, 2021). Chatbots need to be trained how to communicate with users in a natural manner. There are a number of limits to the Thai language. Thai does not have spaces between sentences in its written form, yet same sentences might have multiple meanings based on context, therefore chatbots must interpret the sentence by looking at the previous conversations. However, it's possible to overcome these shortcomings with chatbots equipped with natural language processing (NLP) capabilities (Puglisi, 2019). Currently, Dialogflow supports natural language processing in more than 20 languages including Thai.

According to the information provided above, chatbots are apparently being adopted as an assistant to senior citizens by serving as a personalized tool providing advice through conversations on digital devices, where content options can be added to address the individual needs of senior citizens. In Thailand, in order to address the shortage of personnel with knowledge and expertise in the care and training of specific education for the elderly, chatbots were discovered to be utilized in a number of ways to serve or assist the elderly. "Choojai", for example, is a chatbot that monitors the elderly's negative emotions and analyzes behavioral problems in order to avoid and aid the elderly with sadness, stress, anxiety, and memory issues (SD Thailand, 2019). Besides, chatbots are being used in Thailand to connect online learning with elderly. It primarily encourages elders to pursue lifelong learning on their own and in groups, so assisting in the development of seniors' ability to seek out further information at any time and from any location through the Internet network (Jabjone et al., 2020). Furthermore, "Line" is the best platform for developing chatbots. According to Thailand internet user behavior study in 2019 conducted by Electronic Transactions Development Agency (ETDA), the Line messaging platform is the most widely used online communication application, followed by Facebook Messenger, FaceTime, and WhatsApp (Rodsawang et al., 2020). Nonetheless, no chatbot particularly designed and developed to enhance digital literacy for the senior citizens has been discovered in Thailand.

The main objective of this research is to enhance the digital literacy skills with an intelligent conversational agent, the "Senior See Net" chatbot, for senior citizens in Thailand. To accomplish so, a study of senior citizens in Thailand was conducted to identify their digital literacy level, preferences for user interface design, and expected functionality. Then, depending on the results, we have designed and developed the Senior See Net chatbot with a flow based on such the general chatbot architecture as shown in Fig. 1, the high level of chatbot architecture (Sriwisathiyakun \& Dhamanitayakul, 2021), which Line messaging and API.AI (Dialogflow) were used as the main platform for chatbot development.

\section{Methodology}

This study used research and development approach to investigate baselines on the use of digital platforms of senior citizens in Thailand and innovate the chatbot. This study was conducted in three phases: 
LINE MESSAGING PLATFORM

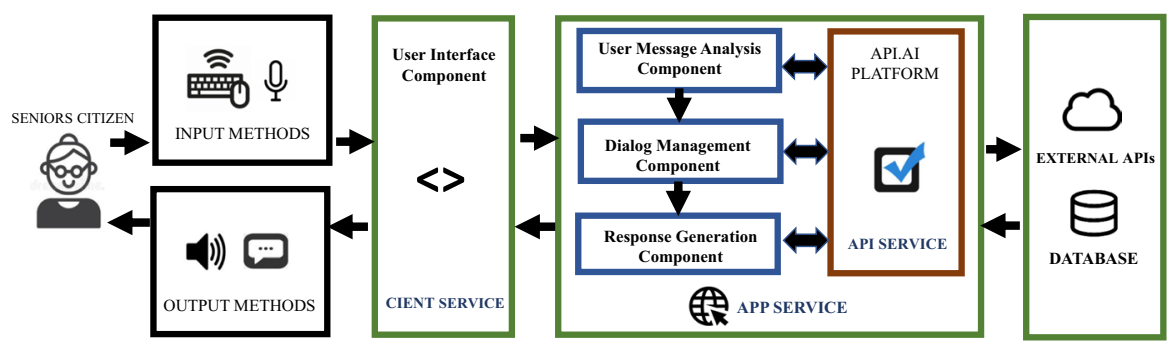

Fig. 1 High level of chatbot architecture

Phase I: The baseline survey was conducted to investigate the use of digital platforms of senior citizens in Thailand There are 11,627,130 senior citizens in the target demographic (Department of Older Persons, 2020). According to Taro Yamane, a sample size of 400 persons was the optimum with a 5\% error. This study collected the data by questionnaires with 422 senior citizens in Bangkok Metropolitan and the six regions of Thailand. A baseline questionnaire on the use of digital platforms of senior citizens in Thailand was employed for the data collection. Five experts assessed this questionnaire for content validity using the Item-Objective Congruence (IOC) index. Its response items were constructed based on a focused group discussion with 10 senior citizens, tested by Cronbach's alpha, and tried out with 30 respondents before the actual survey with 422 samples. In Sect. 1 of Part 4, Results, the survey findings are provided.

Phase II: The chatbot, learning media, and content were developed The survey results of the Phase I were utilized to the design and development of the Senior See Net Chatbot and learning materials. The Software Development Life Cycle (SDLC) approach was applied as a framework for chatbot development, including the stages of Planning, Analysis, Design, Implementation, and Deployment. In Sect. 2 of Part 4 , Results, the details are described.

Phase III: The chatbot, learning media, and content were validated The Senior See Net Chatbot including learning media and content were validated and revised by five experts before further experimentation with senior citizens. Learning media and content for senior citizens were created in interactive messages, videos, and infographics, and validated by experts via a questionnaire. The developed Senior See Net Chatbot was also validated by experts after a pre-experimental evaluation in accessibility, artistic composition, media and content, and functional issues. Expert feedback and suggestions were explored by interviews and used to revise the chatbot before implementing it for senior citizens. The qualitative analysis were used to process the data acquired from the interviews. A conclusion was drawn to describe the overall findings. Among the five experts, two specialists specialized in information technology, one in educational technology, and two in senior citizens. In Sect. 3 of Part 4, Results, the results of validation are presented. 
The questionnaire derived data were analyzed in mean and standard deviation and interpreted with the following mean-range criteria.

4.51-5.00 refers to the extremely high agreement.

$3.51-4.50$ refers to the high agreement.

2.51-3.50 refers to the moderate agreement.

$1.51-2.50$ refers to the low agreement.

1.00-1.50 refers to the extremely low agreement.

\section{Results}

Sect. 1: The collected baseline data on the use of digital platforms of the 422 senior citizens in Thailand are presented in Tables 2, 3, 4, 5 and 6

Table 2 reveals that the majority of the senior citizens were female $(60.66 \%)$ in the age range of 60-69 years (95.02\%) with residential addresses in Bangkok Metropolitan $(71.08 \%)$ and used smartphones $(93.60 \%)$.

Table 3 indicates that most senior citizens used the Line application $(\bar{x}=4.08)$ followed by Youtube $(\overline{\mathrm{x}}=3.71)$ and Facebook $(\overline{\mathrm{x}}=3.61)$.

Table 2 General information

\begin{tabular}{llll}
\hline & DATA & Number & Percentage \\
\hline SEX & Male & 165 & 39.10 \\
& Female & 256 & 60.66 \\
AGE & Transgender & 1 & 0.24 \\
& 60-69 years & 401 & 95.02 \\
& $70-79$ years & 19 & 4.50 \\
REGION & Over 80 years & 0 & 0.00 \\
& Unspecified & 2 & 0.47 \\
& Bangkok & 303 & 71.80 \\
& Central & 51 & 12.09 \\
& Northeast & 21 & 4.98 \\
& South & 11 & 2.61 \\
& North & 24 & 5.69 \\
& East & 6 & 1.42 \\
& West & 4 & 0.95 \\
& Unspecified & 2 & 0.47 \\
SMARTPHONE USE & Yes & 395 & 93.60 \\
& Occasionally & 12 & 2.84 \\
& Never & 0 & 0 \\
& Unspecified & 15 & 3.55 \\
\hline
\end{tabular}

$n=422$. 
Table 3 The top three digital platforms

\begin{tabular}{lllllllll}
\hline Digital platform & \multicolumn{1}{l}{ Level } & \multicolumn{1}{l}{} & & $\bar{X}$ & S.D & Result \\
\cline { 2 - 6 } & Highest & High & Medium & Low & Lowest & & & \\
\hline Line & 204 & 91 & 86 & 36 & 3 & 4.08 & 1.05 & High \\
& $(48.34)$ & $(21.56)$ & $(20.85)$ & $(8.53)$ & $(0.71)$ & & & \\
Youtube & 120 & 126 & 112 & 54 & 8 & 3.71 & 1.07 & High \\
& $(28.57)$ & $(30.00)$ & $(26.67)$ & $(12.86)$ & $(1.90)$ & & & \\
Facebook & 71 & 194 & 96 & 45 & 16 & 3.61 & 1.01 & High \\
& $(16.82)$ & $(45.97)$ & $(22.75)$ & $(10.66)$ & $(3.79)$ & & & \\
\hline
\end{tabular}

$\mathrm{n}=422$.

The digital literacy skills assessed among the senior citizens consisted of the use of digital devices, which here means using a smartphone or tablet computer to Access, Analyze, Evaluate, Participate, and Act. Table 4 suggests that the senior citizens possessed adequate competence to select digital media and applications as their digital literacy skill on Access was rated high $(\overline{\mathrm{x}}=3.78)$. Furthermore, the remaining digital literacy skills (i.e., Analyze - the abilities to analyze and validate facts and news, Evaluate - the assessment of values and benefits in association with selfbenefits and benefits of others, Participate - the use of smartphones or mobile applications to engage in social agendas, and Act-the use of smartphones or mobile applications to create social changes) were rated moderate with mean scores of 3.30 , $3.10,3.32$, and 3.46, respectively.

According to Table 5, when it comes to the designs of UX and UI of the innovation for senior citizens, most of the respondents preferred the use of images along with descriptions $(40.75 \%)$, followed by large fonts $(30.18 \%)$, the use of vivid colors (27.31\%), links to external sources $(4.22 \%)$, and others $(1.54 \%)$, respectively.

Table 6 reveals that most of the respondents preferred the function for interactive communication in digital literacy education $(66.80 \%)$, followed by a function to remind users of upcoming schedules (15.40\%), a function to report security/safety incidents $(9.20 \%)$, a function for instant contacting with responsible agents $(6.60 \%)$, and other functions (1.90\%), respectively.

Sect. 2: Based on these baseline results of how the senior citizens in Thailand used their digital platforms, this study designed and constructed a chatbot and learning media within the Software Development Life Cycle (SDLC) in five steps as follow in Fig. 2:

1. Planning: Relevant tools, technology, and data were extracted from research papers, journals, academic articles to plan, design, and develop the chatbot under the named "Senior See Net".

2. System Analysis: Systems and user preferences were analyzed based on the survey results on the use of digital platforms. The design focus was to address their needs while maintaining the simplicity to understand and operate. According to the results, most senior citizens used Line for messaging and demonstrated 


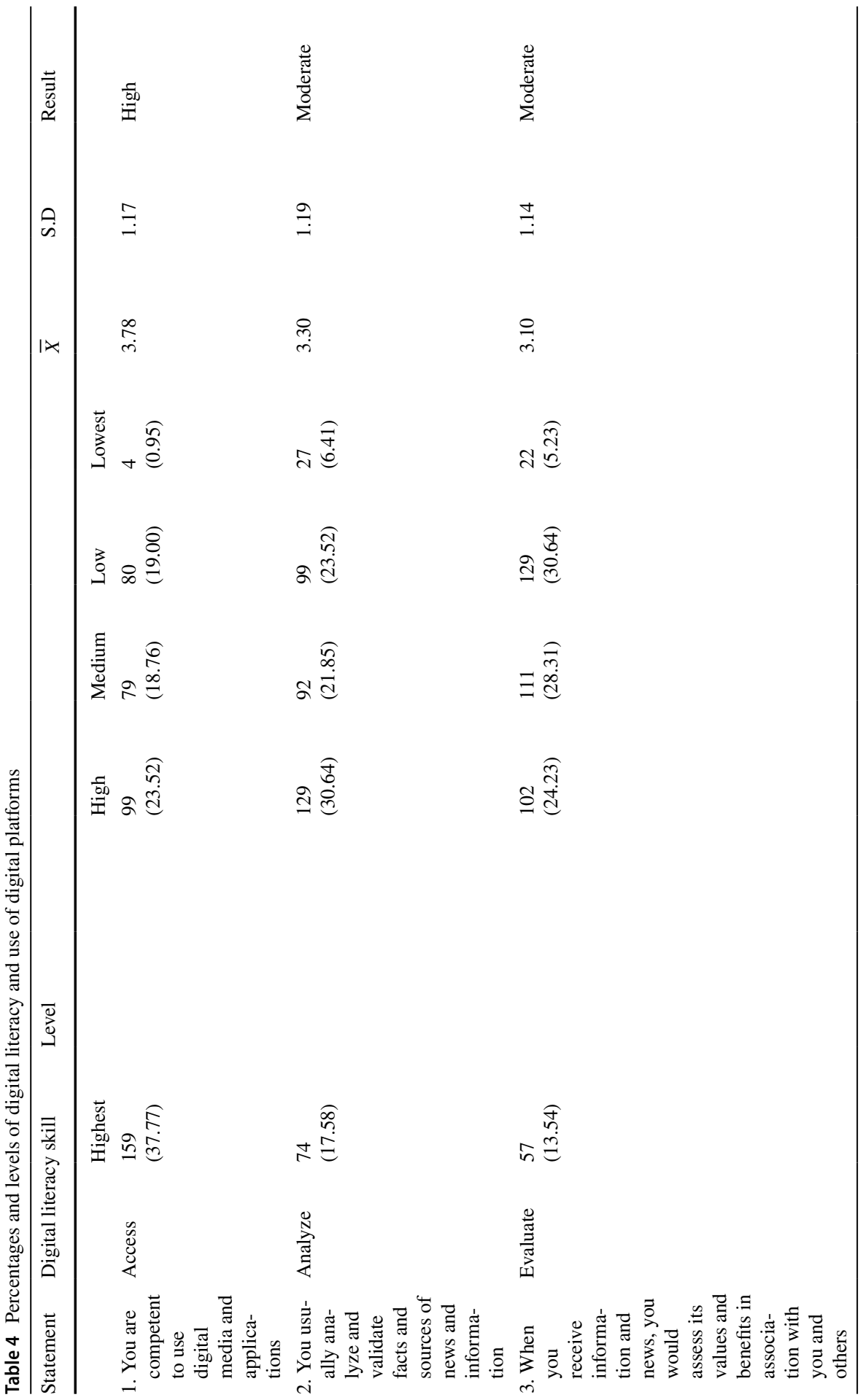




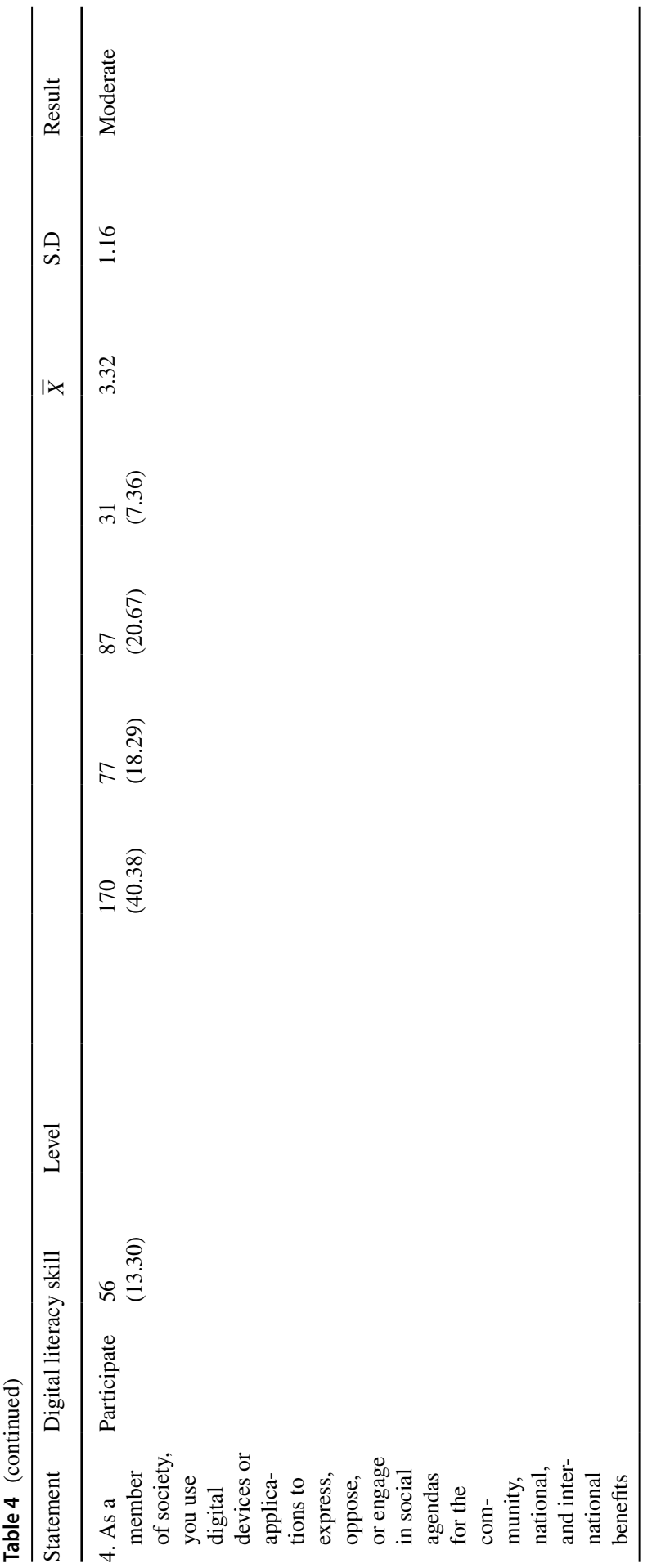




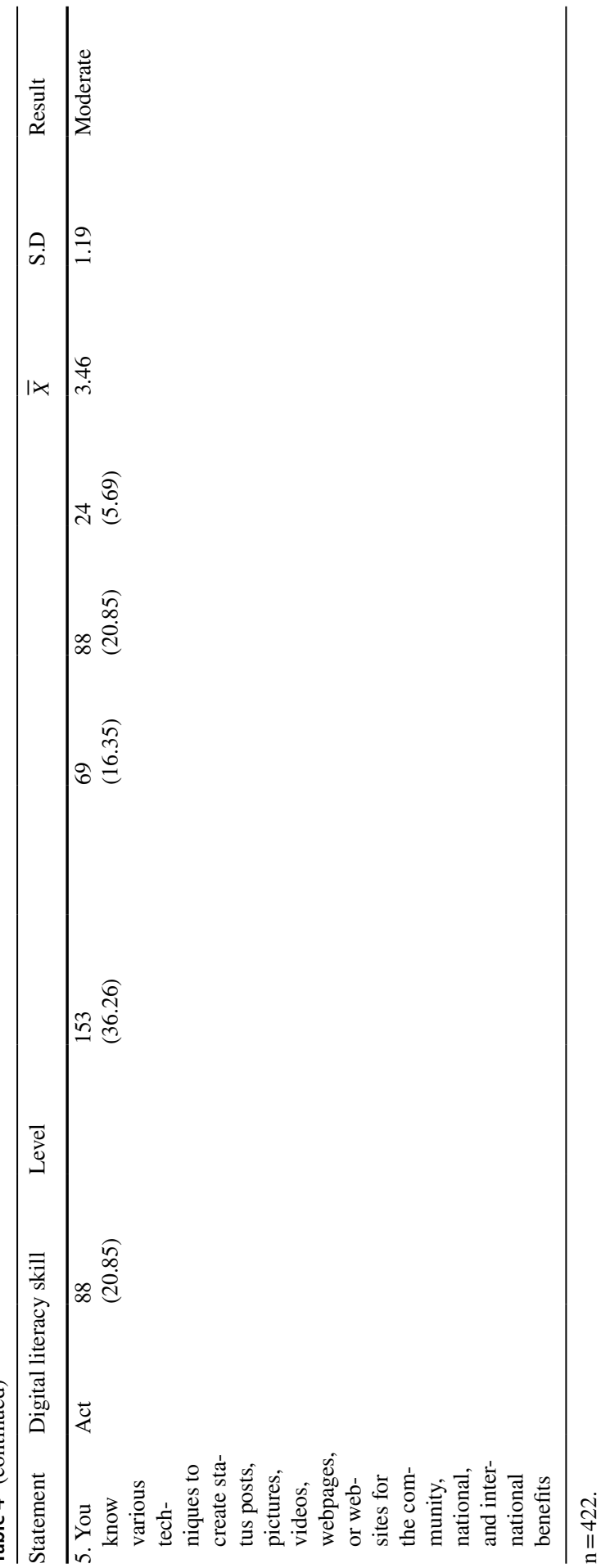

Springer 
Table 5 Preferred UX/UI designs in the innovation for senior citizens

Table 6 Reveals that most of the respondents preferred the service function for interactive

\begin{tabular}{llc}
\hline Design & Number & Percentage \\
\hline Large fonts & 127 & 30.18 \\
Images in descriptions & 172 & 40.75 \\
More vivid colors & 115 & 27.31 \\
Links to external information & 7 & 4.22 \\
$\quad$ sources & 1 & \\
Others & 422 & 1.54 \\
Total & & 100.00 \\
\hline
\end{tabular}

$n=422$.

\begin{tabular}{llc}
\hline Function & Number & Percentage \\
\hline Interactive digital literacy education & 282 & 66.80 \\
Schedule reminders & 65 & 15.40 \\
Security/safety alert & 39 & 9.20 \\
Instant contacting with agents & 28 & 6.60 \\
Others & 8 & 1.90 \\
Total & 422 & 100.00 \\
\hline
\end{tabular}

$\mathrm{n}=422$.

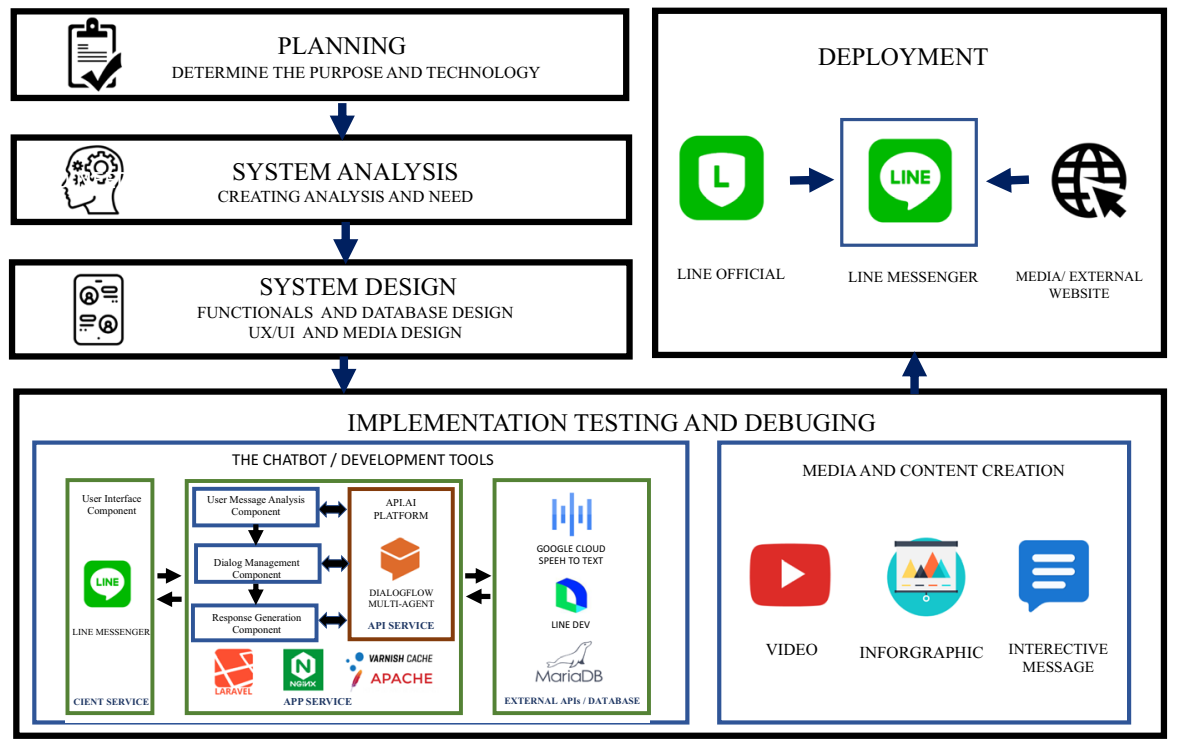

Fig. 2 Chatbot and Learning Media Development framework

high competence in selecting digital media and applications. However, some of their digital literacy skills remained moderate, e.g., the abilities to analyze and validate facts, news, and information sources; and the abilities to operate digital 
devices and applications to engage in social agendas or to create social changes. Therefore, the chatbot was designed to work with the Line messaging platform. Learning Media and content were created in interactive messages, videos, and infographics with functions that are most friendly to senior users.

3. System Design: The system was compartmentally designated in the database, user interface, functionality, media, and content as follow:

3.1 The database to store relevant data was designed to contain six tables in Maria DB, storing schedules, safety alerts, tests, test scores, user data, and user interactions.

3.2 The user interface and service functions were designed based on the survey results in Tables 3, 5, and 6. And the Conversational flow used in the Chatbot was illustrated in Fig. 3.

3.2.1 A Line messenger was designated as the user interface to receive inputs and return outputs between the system and users. This type of interactive solution was conducted through the API service, and application service was required for the operation to address the individual needs of senior citizens.

3.2.2 An interactive menu was designated as a personal-assistant function to provide related information such as emergency phone numbers, online incident reporting, fake news validating, brain training, and recording of schedules. The users also had access to a chat-with-us option should they need to contact real agents.

3.2.3 A user-interaction section was added in the Line messenger with text intent with Line's speech to text option available so that the senior citizens could effortlessly send messages without having to type.

3.3 The digital literacy content and learning media for the senior citizens were designed based on the survey results in Table 4 in interactive messages, videos, and infographics are presented in Fig. 4

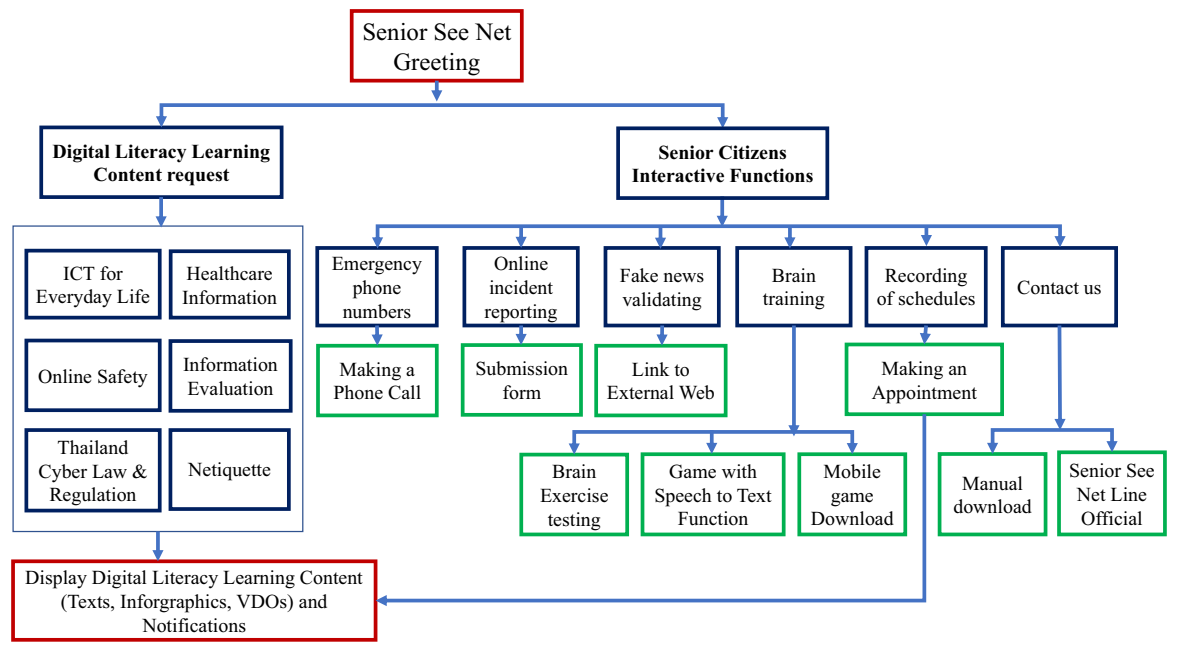

Fig. 3 The Senior See Net Chatbot Conversational Flow 
4. Implement,Testing and Debugging: The Dialogflow (dialogflow.cloud.google. com) was used to develop the designed chatbot system according to the mentioned specifications. Learning media and content were created in interactive messages, videos, and infographics in the interactive display within the chatbot platform as exemplified in Fig. 4. A prototype system was initially developed and evaluated for functionality and responsiveness. System tests and debugs were run to enhance operating stability based on the specifications. The debug mode and logging services were deployed for manual testing in the Laravel framework, allowing messages and system error $\log s$ to be recorded into files. The chatbot was trained to maximize its recognition potential when receiving messages from the users.

5. Deployment: Once the Senior See Net Chatbot, the learning media and content were completely developed. They were submitted to the five experts for a pre-experimental validation and revision. Their feedback was summarized in Sect. 3.

Sect. 3 The Senior See net Chatbot including learning media and content were validated and revised by five experts. The validation results are presented as follows:

Sect. 3.1 The digital literacy content and learning media for the senior citizens were created in interactive messages,videos, and infographics and validated by five experts are presented in Table 7

Table 7 presented the media assessment results by the experts. The overall quality of the media was extremely high $(\bar{x}=4.52)$. Dimensionally, the experts commented that the media were elegantly designed and suitable for the seniors, adequately addressed the digital literacy skills, were seamlessly easy to learn from within the chatbot platform (at the extremely high ratings with the means of 4.80, 4.60, and 4.60 , respectively), contained specific and easily understandable content, and were

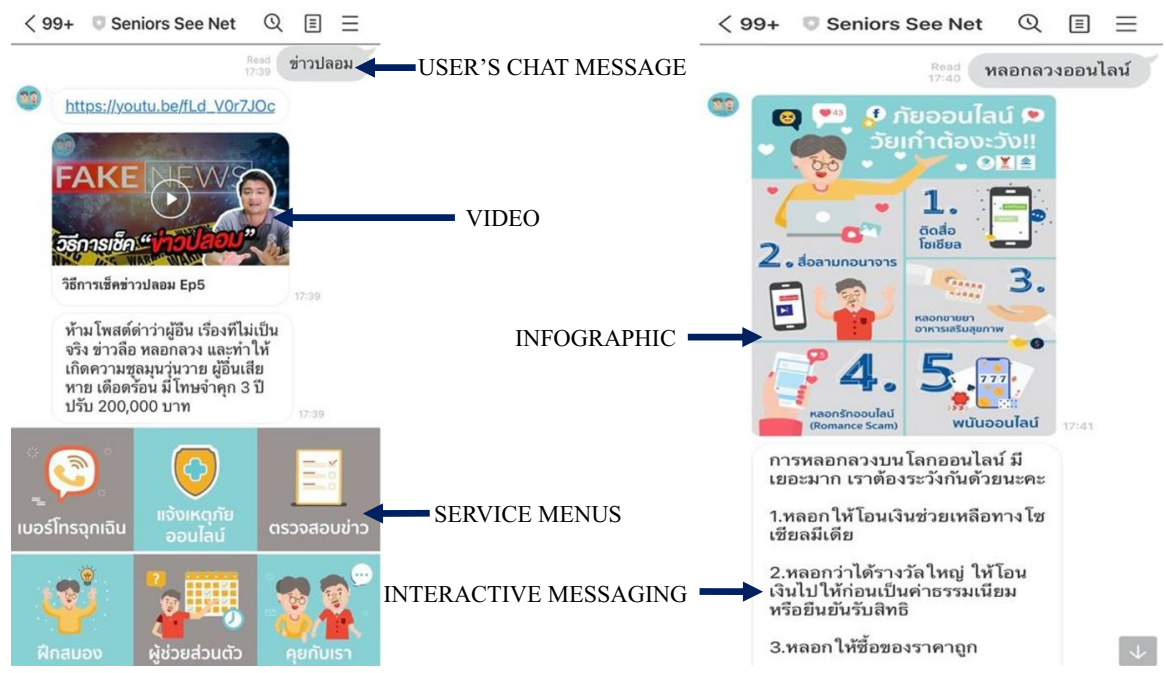

Fig. 4 The Screenshots of user interface and service menus of the chatbot 
Table 7 Learning media and content evaluation by five experts

\begin{tabular}{|c|c|c|c|}
\hline Learning Media Assessment & $\bar{X}$ & S.D & Quality level \\
\hline $\begin{array}{l}\text { The designed instructional media were congruent with the digital literacy } \\
\text { indicators }\end{array}$ & 4.60 & 0.55 & Extremely high \\
\hline $\begin{array}{l}\text { The content of the instructional media was with clarity and easily com- } \\
\text { prehensible }\end{array}$ & 4.20 & 0.45 & high \\
\hline $\begin{array}{l}\text { The instructional media were beautifully designed and tailored to senior } \\
\text { users }\end{array}$ & 4.80 & 0.45 & Extremely high \\
\hline $\begin{array}{l}\text { The users were able to understand the content and learn autonomously } \\
\text { from the media }\end{array}$ & 4.40 & 0.55 & high \\
\hline $\begin{array}{l}\text { The users could seamlessly learn from the media within the chatbot plat- } \\
\text { form without significant issues }\end{array}$ & 4.60 & 0.45 & Extremely high \\
\hline The mean score of the media assessment & 4.52 & & Extremely high \\
\hline
\end{tabular}

$\mathrm{n}=5$.

suitable for autonomous learning (at the high ratings with the means of 4.40 and 4.20 , respectively).

Sect. 3.2 The developed chatbot innovation, in Accessibility, Artistic Composition, Media and Content, and functional issues, validated by five experts and the descriptive summary from the interview are presented as follow:

\subsection{Accessibility}

1. The chatbot was easy to access and utilize.

2. The menu and its functions were easy to operate since they were made of images and texts.

3. All menu items essential for the senior citizens were put together in one place.

\subsection{Artistic composition}

1. The design and choice of colors were appropriate and simplistic.

2. The font size was suitable, considering typical vision challenges that the senior citizens might have.

3. The menu was aligned in the sense that it was friendly to the target group.

\subsection{Media and content}

1. The content was adequately comprehensive in educating digital literacy to the senior citizens. The content was well diversified as it contained texts, infographics, and videos.

2. The content options were plentiful for the senior citizens to access and select as they need. 
3. The content was well organized. Perceivably, the senior citizens should be able to understand, validate, and make decisions from media content and received messages.

4. Games were implemented for users to reviews the knowledge. Associations to relevant external content were present.

\subsection{Functionality}

1. The functions covered all necessary aspects of usage and were suitable for senior users. For instance, the scheduling function was the outstanding one in this application because the senior citizens would be able to plan and schedule the use as they need.

2. Tests and quizzes were present with instant scoring to help the senior citizens review the knowledge and enhance the understanding.

3. The responsiveness of the speech-to-text function was laggy and less than satisfactory.

4. The agent contact function should be revised to ensure that instant interactions between the senior citizens and agents are possible.

\section{Discussion}

The collected baseline data on the use of digital platforms of the 422 senior citizens in Thailand revealed that the majority were female with residential addresses in Bangkok Metropolitan. Furthermore, as many as $93.60 \%$ of them reportedly utilized smartphones, which is in line with the literature review's assertions. This study also discovered that the senior citizens' overall digital literacy was moderate, with "Evaluate" and "Analyze" skills being the lowest compared to "Participate", "Act", and "Access" skills. The notion is congruent with Tayati et al. (2017), stating that senior citizens' overall information and communication technology (ICT) literacy was rated moderate. More specifically, these individuals struggled to analyze and validate online fake news because they lacked the "Analyze" digital literacy skill. Similarly, Takagi et al. (2014) suggested that senior citizens could increase their living quality through information technology (IT). However, the absence of foundation skills caused them to miss too many life opportunities. Consistently, Grigoryeva et al. (2014) found that the senior citizens mostly use the Internet to search for information, read the news, interact with friends, read books and periodicals, and communicate. As a result, it is necessary to develop cybersecurity strategies that are consistent with the behaviours and lifestyles of these senior citizens. BlackwoodBrown (2018) discovered that training is obviously important, as the cybersecurity awareness training was effective in increasing the cybersecurity skill level of the senior citizens, and empowered them with small but significant improvement in the requisite skills to take mitigating actions against cyber attacks.

Another discovery of this study was the fact that Line was the most popular application among the senior citizens, and the notion is in line with Chaichuay 
(2017), who proposed that relevant public agencies should use Line application to help senior citizens enhance their information and media literacy to prevent being victimized by online frauds and scams. As a result, developing a chatbot on the Line platform was a suitable choice. This is in accordance with Rodsawang et al. (2020)'s chatbot design and development. The Line platform was selected to create the "Kor-Ror OK" chatbot, which was designed to support staff by searching for information while staff received calls from people asking questions about COVID-19. Furthermore, it is also in line with Chomsan et al. (2019) who has studied the development of a system for answering inquiries and sending out press releases using chatbots. Customers, particularly the senior citizens, like to communicate using the Line chatbot since it is simple and straightforward to use.

The development of learning content as well as the design of learning media must be based on the results of the survey and analysis of the target audience. In this study, the analyzed baseline data were incorporated in the design and development of the Senior See Net chatbot to enhance the senior citizens' digital literacy that promotes self-paced utilization and autonomous learning. Relevant literature was also reviewed in conjunction with the data to maximize chances to match the needs of the target senior citizens. Based on the survey results, the level of digital literacy of the target senior citizens was used to develop suitable learning media and content. Correspondingly, Chaimaha et al. (2020) developed learning media for the elderly to promote child health in the community based on survey data on the elderly's needs for knowledge. Moreover, this study also found that the senior citizens preferred to have several design features and functions embedded in the innovation. The most wanted design features were the use of images in descriptions, followed by large fonts, while the most preferred function was interactive digital literacy education. This findings are consistent with Farage et al. (2012)'s Design Principles to Accommodate Older Adults, which concludes that simplicity of visual presentation is key: visual clutter should be avoided. Important information should be large, conspicuous, uncrowded, and in the central visual field. Similarly, Ciechanowski et al. (2019) examined affectiveresponse interactions between users and chatbots and found that user interfaces played crucial roles. Their findings show that in interaction with a simpler text chatbot, users had less uncanny effects and negative affect than in cooperation with a more complicated, animated avatar chatbot. The basic chatbot has also resulted in a reduction in the intensity of psychophysiological responses.

After the development of the Senior See Net Chatbot was completed, it was submitted to the experts for further validation. The expert feedback returned positive. Specifically, the chatbot was easy to access, convenient to request for operations, artistically appealing, and comprehensive in content and functionality for enhancing the digital literacy skills. Hence, interacting with the chatbot is projected to yield benefits according to the predetermined goals. As a result of these findings, it is clear that enhancing senior citizens' digital literacy skills is a primary concern. Chatbots are an immensely attractive alternative to bridge the digital literacy gap, since Thailand has attempted to build a nationwide force of digital literacy trainers but has run into a personnel shortage (The Nation Thailand, 2017; SD Thailand, 2019). 


\section{Conclusion}

The main purpose of this research study is to enhance the digital literacy skills of senior citizens in Thailand by using an intelligent conversational agent, the chatbot innovation, as an educational tool. This study investigated the baseline data on the use of digital platforms of the senior citizens in Thailand and discovered that their digital literacy was only rated as moderate. Furthermore, the study also examined the senior citizens' preferences regarding designs for the chatbot innovation. The acquired results were incorporated into the development of the "Senior See Net" chatbot following the Software Development Life Cycle (SDLC). Learning media and content were constructed based on the digital literacy indicators of Access, Analyze, Evaluate, Participate and Act to reinforce the senior citizens' knowledge and ensure that they could maintain safe and secure living in the digital era. Expert feedback revealed that this chatbot was easy to access, convenient to request for operations, artistically appealing, and comprehensive in content and functionality for enhancing digital literacy skills. This Senior See Net chatbot served as an educational assistant to foster digital literacy skills for the senior citizens. It was designed and developed to facilitate the senior citizens with convenient accessibility and learning experiences. It is also noteworthy to mention that introducing a new solution like the chatbot to the senior citizens in Thailand was challenging because such an introduction transformed the way the senior citizens receive education, which had been through a form of interpersonal communication to a new mobile communication approach for enhanced learning autonomy. In the following research sequence, the chatbot was planned to be implemented in realistic situations involving senior citizens to investigate learning outcomes and user satisfaction. To further apply the research results, the chatbot prototype was also planned to be distributed to support agencies across Thailand.

\section{Declarations}

Conflict of Interest There is no conflict of interest.

\section{References}

Adamopoulou, E., \& Moussiades, L. (2020). Chatbots: History, technology, and applications. Machine Learning with Applications. Vol 2. Dec. 2020. https://doi.org/10.1016/j.mlwa.2020.100006.

Almurtadha, Y. (2019). LABEEB: Intelligent Conversational Agent Approach to Enhance Course Teaching and Allied Learning Outcomes Attainment. Journal of Applied Computer Science \& Mathematics, Issue 1/2019, vol.13, No. 27, Suceava. https://doi.org/10.4316/JACSM.201901001.

Blackwood-Brown, C. G. (2018). An Empirical Assessment of Senior Citizens' Cybersecurity Awareness, Computer Self-Efficacy, Perceived Risk of Identity Theft, Attitude, and Motivation to Acquire Cybersecurity Skills. (Research report). Florida: Nova Southeastern University.

Chaichuay, W. (2017). Elderlies' experience in using LINE application: a phenomenological study. Veridian E-Journal, Silpakorn University, Thai edition in the field of humanities: social sciences: and arts., 10,1(January-April 2017), 905-918. 
Chaimaha, N., Putthinoi, S., \& Lersilp, S. (2020). Development of Learning Media for the Elderly to Promote Child Health in the Community. Occupational Therapy International, vol. 2020, Article ID 7252046, 7 pages, 2020. https://doi.org/10.1155/2020/7252046.

Chomsan, C., Nakkrasae, S., \& Sa-ngarmangkang, N. (2019). Question Answering and News Releasing Systems by Chatbot: A Case Study of Ramita Health\&Beauty Co., Ltd. The 9 th STOU National Research Conference. Nonthaburi: Sukhothai Thammathirat Open University. Retrieved from https://www.stou.ac.th/thai/grad_stdy/Masters/\%E0\%B8\%9D\%E0\%B8\%AA\%E0\%B8\%AA/resea rch/9th/STPoster.html

Ciechanowski, L., Przegalinska, A., Magnuski, M., \& Gloor, P. (2019). In the shades of the uncanny valley: An experimental study of human-chatbot interaction. Future Generation Computer Systems, Volume 92, 2019, Pages 539-548, ISSN 0167-739X, https://doi.org/10.1016/j.future.2018.01.055.

Department of Older Persons (2020). Statistics of the elderly in Thailand. [Online]. Retrieved December 13, 2020 from https://www.dop.go.th/th/know/1.

Deursen, A. J.A.M. v., Helsper, E.J., \& Eynon, R. (2016). Development and validation of the Internet Skills Scale (ISS). Information, Communication \& Society. pp. 1-20. ISSN 1369-118X. https://doi. org/10.1080/1369118X.2015.1078834.

Dhamanitayakul, C. (2017). Conceptualization Digital Citizenship for Digital Natives in Thailand. (Research report). Bangkok: National Institute of Development Administration.

Farage, M., Miller, K., Ajayi, F., \& Hutchins, D. (2012). Design Principles to Accommodate Older Adults. Global Journal of Health Science, 4(2), 2-25. https://doi.org/10.5539/gjhs.v4n2p2

Friemel, T. N. (2016). The digital divide has grown old: Determinants of a digital divide among seniors. New Media \& Society, 18, 313-331. https://doi.org/10.1177/1461444814538648

Google Cloud. (2021). Dialogflow. [Online]. Retrieved November 13, 2021 from https://cloud.google. com/dialogflow

Grigoryeva, I., Shubinskiy, M., \& Mayorova, E. (2014). ICT as a driver for senior citizens' social inclusion. ICEGOV2014: 8h $^{\text {th }}$ International Conference on Theory and Pracice of ElectroniGovernace Guimaraes Portugal. 292-295. https://doi.org/10.1145/2691195.2691260.

Hoblert, S., \& Wolff, R.M.v. (2019). Say Hello to Your New Automated Tutor - A Structured Literature Review on Pedagogical Conversational Agents. 14th International Conference on Wirtschaftsinformatik. Siegen, Germany. Retrieved from https://aisel.aisnet.org/wi2019/track04/papers/2/

Jabjone, S., Lowanichai, S., Puttasimma, T., \& Jongmuanwai, B. (2020). Developing Media of Chatbot Online Learning for Elderly. Journal of Technology Management Rajabhat Maha Sarakham University, 7(2), 47-58. Retrieved from https://ph02.tcithaijo.org/index.php/itm-journal/article/view/ 241426

Kitchenham, B. (2004). Procedures for Performing Systematic Reviews (p. 33). UK, Keele University.

Knodel, J., Prachuabmoh, V., \& Chayovan, N. (2013). The Changing Well-Being of Thai Elderly: An Update from the 2011 Survey of Older Persons in Thailand (Research Report). Chiang Mai: HelpAge International.

Kompella, R. (2018). Conversational AI chat-bot-Architecture overview. [Online]. Retrieved October 18, 2021 from https://towardsdatascience.com/architecture.overview-of-a-conversational-ai-chat-bot4ef3dfefd52e.

Liao, Q.V., Hussain, M.M., Chandar, P., Davis, M., Khazaeni, Y., Crasso, M.P., Wang, D., Muller, M.J., Shami, N.S., \& Geyer, W. (2018). All Work and No Play? Conversations with a Question-andAnswer Chatbot in the Wild. Proceedings of the 2018 CHI Conference on Human Factors in Computing Systems. Association for Computing Machinery, New York, NY, USA, Paper 3, 1-13. https:// doi.org/10.1145/3173574.3173577

Luxton D.D., \& Sirotin A. (2020). Intelligent Conversational Agents in Global Health. In: Okpaku S. (eds) Innovations in Global Mental Health. Springer, Cham. https://doi.org/10.1007/978-3-31970134-9_11-1

Mislevics, A., Grundspenţis, J., \& Rollande, R. (2018). Systematic approach to implementing chatbots in organization - RTU Leo showcase. BIR Workshops. http://ceur-ws.org/Vol-2218/paper36.pdf.

Oxford Dictionary (2019). Chatbots. [Online]. Retrieved October 18, 2021 from https://en.oxforddict ionaries.com/definition/chatbot.

Pérez-Marín, D. (2021). A Review of the Practical Applications of Pedagogic Conversational Agents to Be Used in School and University Classrooms. Digital, 2021(1), 18-33. https://doi.org/10.3390/ digital1010002 
Puglisi, G. (2019). Chatbots find fertile ground in Thailand, Southeast Asia's largest social commerce market. [Online]. Retrieved November 13, 2021 from https://kr-asia.com/chatbots-find-fertileground-in-thailand-southeast-asias-largest-social-commerce-market.

Rodsawang, C., Thongkliang, P., Intawong, T., Sonong, A., Thitiwatthana, Y., \& Chottanapund, S. (2020). Designing a Competent Chatbot to Counter the COVID-19 Pandemic and Empower Risk Communication in an Emergency Response System. Outbreak, Surveillance, Investiagtion \& response (OSIR) Journal, 13(2), 71-77.

Schreurs, K., Quan-Haase, A., \& Martin, K. (2017). Problematizing the digital literacy paradox in the context of older adults' ICT use: Aging, media discourse, and self-determination. Canadian Journal of Communication, 42(2). https://doi.org/10.22230/cjc.2017v42n2a3130

SD Thailand. (2019). Mahidol creates "Choo Jai," a prototype robot that can assist in the mental care of the elderly. [Online]. Retrieved October 18, 2021 from https://sdthailand.com/2019/11/mahidoldevelop-chujai-robot-for-elderly-people.

Shevat, A. (2017). Designing bots: Creating conversational experiences. O'Reilly Media Inc.

Sindecharak, T., \& Kwanyoo, A. (2020). The demand for information technology and digital communications among the elderly. Silpakorn University Journal: SUJ, 40(3), 75-96. https://doi.org/10.14456/ sujthai.2020.24

Song, D., Oh, E.Y., \& Rice, M. (2017). Interacting with a conversational agent system for educational purposes in online courses. The10th International Conference on Human System Interactions (HSI). https://doi.org/10.1109/HSI.2017.8005002.

Sriwisathiyakun, K., \& Dhamanitayakul, C. (2021). Innovation for Digital Citizenship Skill Development: Cyber Security in Information Technology for Senior Citizen (Research report). Bangkok: National Broadcasting and Telecommunication Commission.

Sultana, T.N. (2018) . Digital literacy Among Muslim Women Study of Vijayapur city. International Journal of Creative Research Thoughts (IJCRT), 6(1), 1424-1432. Retrieved from https://www.ijcrt. org/papers/IJCRT1705204.pdf

Takagi, H., Kosugi, A., Ishihara, T., \& Fukuda, K. (2014). Remote IT education for senior citizens. Proceedings of the 11th Web for All Conference (W4A '14). Association for Computing Machinery, New York, NY, USA, Article 41, 1-4. https://doi.org/10.1145/2596695.2596714

Tayati, P., Disathaporn, C., \& Onming, R. (2017). The Model of Thai Elderly Learning Management for Information and Communication Technology Literacy. Veridian E-Journal, Silpakorn University, 10(3). Retrieved from https://he02.tcithaijo.org/index.php/Veridian-E-Journal/article/view/106455/ 84282

Techataweewan, W., \& Prasertsin, U. (2018). Development of digital literacy indicators for Thai undergraduate students using mixed method research. Kasetsart Journal of Social Sciences, Volume 39, Issue 2, 2018, Pages 215-221. ISSN 2452-3151. https://doi.org/10.1016/j.kjss.2017.07.001.

The Nation Thailand. (2017). 1,000 digital literacy trainers to bridge gap. [Online]. Retrieved October 18, 2021 from https://www.nationthailand.com/Startup_and_IT/30328563.

Vaidyam, A.N., Wisniewski, H., Halamka, J.D., Kashavan, M.S., \& Torous, J.B. (2019). Chatbots and Conversational Agents in Mental Health: A Review of the Psychiatric Landscape. Can J Psychiatry. 2019 Jul;64(7):456-464. https://doi.org/10.1177/0706743719828977

Winkler, R., Hoblet, S., Salovaara, A., \& Sollner, M. (2020). Sara, the Lecturer: Improving Learning in Online Education with a Scaffolding-Based Conversational Agent. CHI '20: Proceedings of the 2020 CHI Conference on Human Factors in Computing Systems.pp. 1-14. https://doi.org/10.1145/ 3313831.3376781.

Wiratunga, N., Cooper, K., Wijekoon, A., Palihawadana, C., Mendham, V., Reiter, E., \& Martin, K. (2020). FitChat: Conversational Artificial Intelligence Interventions for Encouraging Physical Activity in Older Adults. arXiv preprint arXiv:2004.14067. Retrieved from https://arxiv.org/pdf/ 2004.14067.pdf

Wollny, S., Schneider, J., Di Mitri, D., Weidlich, J., Rittberger, M., \& Drachsler, H. (2021). Are We There Yet? - A Systematic Literature Review on Chatbots in Education. Front. Artif. Intell., 4, 654924. https://doi.org/10.3389/frai.2021.654924

Publisher's Note Springer Nature remains neutral with regard to jurisdictional claims in published maps and institutional affiliations. 